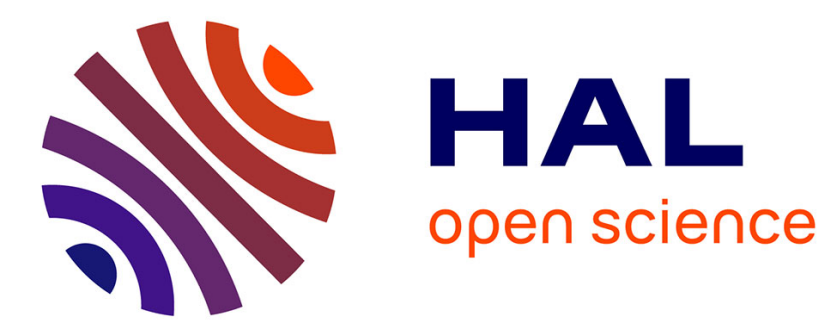

\title{
Penser le droit à la santé au prisme des capacités de base
}

Caroline Guibet Lafaye

\section{To cite this version:}

Caroline Guibet Lafaye. Penser le droit à la santé au prisme des capacités de base. Jean-Marc Ferry;

Caroline Guibet Lafaye; Mark Hunyadi. Penser la santé, PUF, pp.55-88, 2009. hal-00357630

\section{HAL Id: hal-00357630 \\ https://hal.science/hal-00357630}

Submitted on 30 Jan 2009

HAL is a multi-disciplinary open access archive for the deposit and dissemination of scientific research documents, whether they are published or not. The documents may come from teaching and research institutions in France or abroad, or from public or private research centers.
L'archive ouverte pluridisciplinaire HAL, est destinée au dépôt et à la diffusion de documents scientifiques de niveau recherche, publiés ou non, émanant des établissements d'enseignement et de recherche français ou étrangers, des laboratoires publics ou privés. 


\title{
Penser le droit à la santé au prisme des capacités de base
}

\author{
Caroline Guibet Lafaye
}

Centre Maurice Halbwachs

CNRS

48, bd Jourdan, F-75014 Paris (France)

caroline.guibet-lafaye@ens.fr

\section{Introduction : « La santé pour tous en l'an 2000 »}

En 1977, la trentième Assemblée mondiale de la santé a fixé à l'OMS comme objectif prioritaire de «faire accéder d'ici l'an 2000 tous les habitants de la planète à un niveau de santé qui leur permette de mener une vie socialement et économiquement productive », sachant, par ailleurs, que l'OMS définit la santé comme un «état de complet bien-être physique, mental et social» (OMS, 1948). L'objectif de «la santé pour tous » est une interprétation de l'article premier de la constitution de l'OMS stipulant qu'il faut «amener tous les peuples au niveau de santé le plus élevé possible». On trouve également, dans les politiques communautaires, à partir de l'Acte Unique de 1986, la volonté d'assurer un haut niveau de protection de la santé.

La stratégie mondiale de « la santé pour tous d'ici l'an $2000 »$ - adoptée à l'unanimité par l'Assemblée mondiale de la santé en 1981 - a pour axes principaux l'équité et le développement des soins primaires ${ }^{1}$, par la mise en place progressive de programmes de

\footnotetext{
${ }^{1}$ «On entend par soins de santé primaires des soins de santé essentiels universellement accessibles à tous les individus et à toutes les familles de la communauté par des moyens qui leur sont acceptables, avec leur pleine participation et à un coût abordable pour la communauté et le pays. Les soins de santé primaires font partie intégrante du système de santé du pays, dont ils constituent le noyau, ainsi que du développement social et économique global de la communauté » (Définition de l'OMS).
} 
santé, touchant l'ensemble des populations. Plus près de nous, les 191 Etats membres des Nations Unies s'engageaient, par la Déclaration des objectifs du Millénium, à « réduire de moitié, entre 1990 et 2015, la proportion de la population dont le revenu est inférieur à un dollar par jour» (Objectif 1.A). De même, le «Deuxième programme d'action communautaire [européen] dans le domaine de la santé (2008-2013)» affiche la volonté de réduire les écarts de santé 1 .

Les soins de santé primaires, dans la définition qu'en propose l'OMS, ont pour vocation de maîtriser les principaux problèmes de santé et revêtent diverses formes : actions de promotion, de prévention, de soins curatifs et de réadaptation. En tant qu'ils reflètent et procèdent des conditions économiques et des valeurs sociales des pays et de leurs communautés constitutives, ils varient d'un pays et d'une communauté à l'autre mais ils comprennent, au minimum, les activités suivantes: promotion d'une nutrition correcte et d'un approvisionnement convenable en eau saine; mesures d'assainissement de base; santé maternelle et infantile, y compris planification familiale; vaccination contre les grandes pathologies infectieuses ; prévention et réduction des endémies locales ; éducation concernant les problèmes de santé prédominants et les moyens de les prévenir et de les maîtriser; traitement approprié des maladies et des traumatismes courants ${ }^{2}$.

Pourtant ni dans les objectifs du Millénium ni dans la politique européenne commune, le souci pour la santé ne constitue une priorité alors même qu'au niveau mondial, un tiers des décès annuels, c'est-à-dire 18 millions de personnes par an, est dû à des causes liées à la pauvreté aisément évitables par une meilleure alimentation, de l'eau potable, des solutions économiques de réhydratation, des vaccins et des anti-biotiques ou d'autres médicaments de base. La réflexion que nous proposons consistera à montrer pourquoi l'on ne peut s'en remettre aux objectifs du Millénium pour que soit réalisée « la santé pour tous » ou « assur[é] un haut niveau de protection de la santé » dans l'Union Européenne, à partir (1) d'une argumentation fondée sur la validité, au plan global, des théories de la justice convoquées, pour défendre la priorité ou la relativité des soins en santé primaires ; à partir (2) de la mise évidence des fondements éthiques sur la base desquels une définition et une politique des biens premiers en santé sont formulées. (3) Enfin nous montrerons que le souci pour la pauvreté et la volonté de garantir la santé pour tous gagneraient tous deux à concevoir les

\footnotetext{
1 Décision $n^{\circ}$ 1350/2007/CE du Parlement européen et du Conseil du 23 octobre 2007 établissant un deuxième programme d'action communautaire dans le domaine de la santé (2008-2013).

${ }^{2}$ Spécialement dans les pays en développement, le développement économique, les actions de lutte contre la pauvreté, la production alimentaire, l'eau, l'assainissement, le logement, la protection de l'environnement et l'éducation concourent tous à la santé et ont pour objectif commun le développement humain.
} 
biens premiers en santé, non pas seulement à partir d'une interprétation des besoins fondamentaux, mais surtout à partir d'une référence aux capacités (ou capabilités), tels qu'A. Sen ou M. Nussbaum les envisagent.

Cette approche vise à inspirer la nouvelle orientation de la politique européenne de santé publique et prétend pouvoir répondre plus adéquatement à la volonté, affirmée par l'Union Européenne d' " agir sur le long terme », en l'occurrence sur « les déterminants de santé », en formulant des objectifs de santé non plus seulement en termes de biens primaires - inspirés de la philosophie rawlsienne et que l'on trouvait dans la Charte Sociale des droits fondamentaux de 1989 de l'UE $^{1}$ à travers l'accès à la prévention et aux soins curatifs - mais en termes de capacités.

\section{Le sens de la priorité des soins en santé : la santé, une « sphère séparée de la justice »?}

Ce statut, dans une certaine mesure, second de la santé, dans les priorités de justice globale du Millénaire et dans celles de la politique européenne commune, se comprend - en particulier dans le premier cas - lorsque l'on envisage les théories de la justice implicitement convoquées ainsi que celles qui motivent les objectifs de l'OMS. Les objectifs du Millénium sont au nombre de huit: 1. Réduire l'extrême pauvreté et la faim ; 2. Assurer l'éducation primaire pour tous ; 3. Promouvoir l'égalité des sexes et l'autonomisation des femmes ; 4 . Réduire la mortalité des enfants de moins de 5 ans; 5 . Améliorer la santé maternelle; 6. Combattre le VIH/sida, le paludisme et d'autres maladies; 7. Assurer un environnement durable ; 8 . Mettre en place un partenariat mondial pour le développement.

Le fait de conférer une priorité à la réduction de la pauvreté, évaluée à partir des revenus (puisqu'il s'agit de réduire de moitié, d'ici 2015, le nombre de personnes vivant avec moins de 2 dollars par jour), repose sur une conception de la justice « unitaire » («one-justice ») ou encore globale plutôt que sur une conception de la justice, articulée en « sphères » (« unified justice »), pour reprendre la distinction utilisée par S.-C. Kolm, dans son article « On Health and Justice $»^{2}$.

\footnotetext{
${ }^{1}$ Charte Sociale des droits fondamentaux, article $35:$ :Toute personne a le droit d'accéder à la prévention en matière de santé et de bénéficier de soins médicaux dans les conditions établies par les législations et pratiques nationales. Un niveau élevé de protection de la santé humaine est assuré dans la définition et la mise en œuvre de toutes les politiques et actions de l’Union ».

2 S.-C. Kolm, «On Health and Justice », Institute for Advanced Studies in the Social Sciences, Paris, Février 2002. Disponible à l'adresse :

http://mora.rente.nhh.no/projects/EqualityExchange/Archiveoldermanuscripts/tabid/66/Default.aspx
} 
Plus exactement, deux conceptions globales de la justice sont convoquées dans la description des objectifs du Millénium : d'une part, une conception de la justice globale, dans la mesure où la réduction de la pauvreté est la priorité absolue et, d'autre part, une conception de la justice fondée sur des sphères, au sein desquelles l'optimum doit, à chaque fois, être recherché : les objectifs du Millénium sont effectivement déclinés en huit domaines s'articulant autour de la pauvreté, de l'éducation, de l'égalité, de la santé et de l'environnement.

Or la question de la justice distributive s'ordonne, communément, autour de trois problématiques : (a) les niveaux de la justice, (b) le choix entre la responsabilité individuelle et la responsabilité collective, (c) la rationalité de l'équité. L'articulation de ces niveaux de la justice présuppose une orientation théorique préliminaire, quant à l'interprétation même du concept de justice dont on doit établir si elle est une - i.e. appréhendée globalement - ou bien si elle doit être considérée comme l'unification de plusieurs sphères de justice distinctes, telles que la santé, l'éducation ou les ressources, la consommation courante ou encore les services judiciaires et postaux ${ }^{1}$. Dans le second cas, tous les secteurs de la justice sont envisagés conjointement, dans leur capacité à se compenser mutuellement, dans la mesure où une personne peut, par exemple, être mal pourvue dans un domaine sans l'être dans un autre. A l'inverse, lorsque l'on considère que la distribution du revenu et de la richesse est première et essentielle, les «sphères » de la justice sont envisagées différemment. En particulier une solution consiste à réaliser, en priorité, la macrojustice, en l'occurrence une distribution du revenu optimum, et à laisser ensuite les questions spécifiques de justice être déterminées par un processus de laissez faire, c'est-à-dire par des actions libres, par le marché, par des échanges et des accords. Si dans l'un de ces mécanismes advient un échec, dû à un manque de moyens, à un manque d'information ou au coût d'une exclusion, on essaie, à travers le recours au secteur public, de réaliser ce que cette action ou cet échange serait parvenu à faire aboutir en cas de succès ${ }^{2}$.

\footnotetext{
${ }^{1}$ L'idée étant que l'on cherchera alors la façon optimale de concevoir, en termes d'efficacité redistributive, la justice dans chaque sphère.

${ }^{2}$ See Kolm 1985, 1987a, 1987b, 1996. La politique actuelle de santé au niveau européen s'inscrit dans cette conception des sphères de la justice. La santé est spécifiquement envisagée comme un enjeu transversal pouvant potentiellement concerner n'importe quelle politique communautaire. Ainsi le Traité de Maastricht souligne que «les exigences en matière de protection de la santé sont une composante des autres politiques de la Communauté » (Traité de Maastricht, 1992, article 129).

Bien que la réduction des écarts de santé, dont on s'accorde à reconnaître qu'ils sont très importants en particulier concernant l'espérance de vie à la naissance et la prévalence de nombreuses pathologies graves, reste l'un des objectifs fondamentaux de la politique européenne, l'Union n'agit qu'en complément de l'action des différents Etats et s'en tient à une politique de santé très localisée. Dans le programme de santé publique (20032008), adopté le 23 septembre 2002 et doté d'un budget de plus de 300 millions d'euros, conçu comme
} 
L'insistance portée sur la réduction de moitié de la pauvreté dans le monde, au titre d'objectif premier du Millénium, semble répondre à une interprétation de la justice comme justice globale. Cette orientation a pour effet de rendre non pertinente toute considération de justice dans un domaine spécifique, i.e. de façon isolée, y compris s'agissant de la santé. En d'autres termes, on juge que la réduction de la pauvreté permettra, par elle-même, de rendre accessibles à davantage de personnes les soins en santé.

L'un des arguments expliquant que l'on néglige la justice distributive dans les sphères spécifiques de la justice est que celle-ci pourrait ne pas être réalisée et qu'alors les politiques sectorielles (en éducation, santé, environnement par exemple) devraient elles-mêmes contribuer, dans la mesure de leurs moyens, à la réalisation de la justice globale à travers et grâce à des mesures distributives spécifiques, i.e. prises au sein de ces sphères elles-mêmes. Pourtant on ne peut moralement défendre l'idée qu'il faille attendre de la réduction mondiale de la pauvreté qu'elle résolve, par elle seule, les problèmes de santé dans le monde. Des mesures spécifiques, notamment de justice distributive, doivent être prises dans le secteur de la santé également.

Une autre raison justifiant de considérer la justice à partir de sphères spécifiques - comme le voudrait la priorité conférée à la politique de santé de l'OMS sur tout autre objectif de justice globale - est que les revendications en matière de justice se formulent communément à partir de ces sphères, i.e. que les personnes elles-mêmes ont tendance à les envisager de cette façon ${ }^{1}$. Dans cette perspective, l'idéal de la justice se formule alors comme un accès égal de chacun à ces biens spécifiques.

En outre la santé, y compris appréhendée comme une "sphère de la justice », a un statut particulier. D'une part, les besoins de base en santé ne sont pas substituables à d'autres types de biens, comme les biens de consommation. D'autre part, il y a des situations, notamment lorsqu'il s'agit d'établir des priorités, où la santé doit être considérée de façon indépendante, i.e. pour elle-même, sans qu'elle puisse entrer dans aucune considération de substituabilité avec d'autres sphères de la justice. Ainsi la santé est et doit être prioritaire, lorsque peu de ressources lui sont consacrées et que les autres aspects de la vie ne sont pas aussi mal servis, ou bien encore lorsque tous les besoins essentiels des populations sont mesurés en rapport à la santé, c'est-à-dire lorsque la famine, la malnutrition, l'insuffisance alimentaire, le manque de

complétant les politiques nationales, les objectifs se résumaient à : (a) améliorer l'information et les connaissances en vue d'une meilleure santé publique ; (b) renforcer la capacité à réagir rapidement et de manière coordonnée aux menaces pour la santé ; (c) promouvoir la santé et prévenir les maladies en prenant en compte les facteurs déterminants pour la santé à travers toutes les politiques existantes.

${ }^{1}$ Voir M. Walzer, Spheres of Justice, New York, Basic Books ; Oxford, Martin Robertson, 1983. Voir aussi, pour un raisonnement comparable Max Weber. 
logement, etc. sont mesurés par les effets néfastes qu'ils produisent sur la santé. La satisfaction de certains besoins de base de santé est alors nécessaire - et par conséquent prioritaire -, pour apprécier tout autre type de consommation. Dans ce cas, la substituabilité est faible et la satisfaction de ces besoins en santé doit être traitée comme une «sphère séparée de justice $»$.

Pour ces raisons, on ne peut considérer que la réduction de la pauvreté doive nécessairement primer sur l'accès de tous aux soins de santé primaires ni qu'il faille privilégier les objectifs du Millénium au regard de ceux de l'OMS. En d'autres termes la priorité, caractéristique de la santé, signifie et a pour conséquence que la substituabilité avec d'autres biens devrait être limitée ${ }^{1}$. Puisque la santé est le premier des besoins de base, la limitation d'un certain nombre de risques sanitaires constitue une priorité. Cet objectif est précisément ce que vise à réaliser la politique des soins primaires en santé, conçue par l'OMS à partir de la fin des années 1970. La notion de soins primaires suppose une distinction précisément établie entre ces derniers et tout autre type de consommation, cette distinction reposant sur des valeurs implicites relatives à l'appréhension et à la définition même de la santé. De ce point de vue, la politique des biens primaires en santé de l'OMS semble plus pertinente que les objectifs 4 à 7, proclamés dans la déclaration du Millénium. En effet, les besoins de base en nourriture et en logement doivent être satisfaits pour éviter des risques sanitaires de malnutrition ou des risques sanitaires dus au climat, alors même que ce type d'exigences ne figure que dans les préoccupations les plus lointaines du Millénaire.

\section{Fondements éthiques de la priorité des soins primaires en santé}

\subsection{Les besoins de base : des besoins fondamentaux et prioritaires}

La priorité fondamentale à accorder à une politique garantissant à tous des biens premiers en santé induit, à l'égard de la communauté - dont il faudra préciser les contours -, un devoir moral. Bien que des auteurs comme M. Walzer défendent l'idée qu'aucune postulation $a$ priori de la nature des besoins n'est possible, on admet que certains besoins tombent, sans ambiguité, du côté des «besoins de base », alors que d'autres restent à la frontière de ces

\footnotetext{
${ }^{1}$ Remarquons que les objectifs de santé décrits dans la déclaration du Millénaire (4. Réduire la mortalité des enfants de moins de 5 ans ; 5 . Améliorer la santé maternelle ; 6 . Combattre le VIH/sida, le paludisme et d'autres maladies ; 7. Assurer un environnement durable) sont moins fondamentaux que ceux visés par l'OMS, dans sa politique de soins primaires en santé.
} 
derniers et des besoins qui ne sont pas fondamentaux. Les biens premiers relatifs à la santé font partie des besoins humains, c'est-à-dire des besoins que les personnes ont parce qu'elles sont des êtres humains, quels que soient leurs objectifs ou leurs fins ${ }^{1}$. Ces besoins recouvrent ce qui est nécessaire pour survivre, pour être en bonne santé, pour éviter la souffrance, pour vivre correctement.

Ces besoins prioritaires et objectifs servent de support de comparaison et d'évaluation, dans des discours prescriptifs ou des choix collectifs concernant des allocations de moyens ${ }^{2}$. Or la satisfaction de ces besoins, susceptibles d'être évalués objectivement, est précisément ce que requiert l'exigence de "santé pour tous ». Ces besoins tiennent lieu de norme sociale, et en l'occurrence de norme globale de santé. En ce sens, le concept de "santé » renvoie inévitablement à une construction sociale normative, qui indique un certain degré de disponibilité globale des conditions favorables internes, externes et apparentées dans la vie humaine. Une théorie morale, à tout le moins une dimension normative, occultée dans leur définition courante, est sous-jacente à la détermination même des biens premiers en santé.

Alors que les besoins instrumentaux, en tant que tels, n'ont pas de poids moral, les besoins de base, en tant qu'ils sont premiers, ont un sens moral. [Ainsi des notions telles que la santé, la souffrance et le fonctionnement correct du corps et de l'esprit ont un statut normatif, pour toute revendication concernant les besoins de base.] Ce statut justifie que les besoins de base imposent des devoirs, en ce sens que ma maladie conditionne une revendication légitime à l'égard d'autrui que mes caprices, mes désirs, mes plaisirs et même mon bonheur ne motivent pas légitimement. Ces revendications s'imposent d'autant plus qu'elles ne dépendent pas de souhaits ou de buts contingents mais s'enracinent dans la nature humaine ${ }^{3}$.

Dans cette mesure, l'institution d'un objectif tel que « la santé pour tous en l'an 2000 », n'était pas un objectif arbitraire ou conventionnel mais constituait bien plutôt l'expression d'une obligation morale. Dans cette perspective, il est essentiel que la notion de « besoins de base » ne demeure pas indéterminée car elle ne pourra, le cas échéant, fonder aucune exigence éthique à l'égard d'autrui, aucun devoir. En effet si le mal, la souffrance, la maladie, la déficience étaient des questions conventionnelles ou des déterminations arbitraires, les

\footnotetext{
${ }^{1}$ Voir J. Griffin, Well-being. Its Meaning, Measurement and Moral Signifiance, Oxford Clarendon Press, 1986, p. 41.

${ }^{2}$ Néanmoins la question des besoins et des besoins premiers ne se pose pas de façon identique selon que l'ensemble des membres d'une société a ou non accès à l'eau potable, ou selon qu'il s'agit ou non d'une société industriellement avancée comme la nôtre.

${ }^{3}$ Nous verrons ultérieurement que l'on peut proposer à partir de là une définition du « bien-être, c'est-à-dire au moins la conception du bien-être, qui doit être utilisée comme une mesure interpersonnelle du jugement moral, [qui] est le niveau auquel les besoins de base sont satisfaits » (J. Griffin, Well-being, p. 42).
} 
devoirs et engagements les concernant demeureraient également arbitraires ou conventionnels.

\section{2 «La santé pour tous » : un devoir moral de la communauté}

a) Détermination impersonnelle des biens premiers en santé

Cette détermination des biens premiers en santé, motive une obligation de la communauté à l'égard de ses membres, en l'occurrence des devoirs, incombant à la communauté internationale et qui pourrait également être ceux de l'Union européenne. L'exigence morale de garantir «la santé pour tous » se justifie, en premier lieu, du fait que l'identification de besoins fondamentaux en santé n'est possible qu'à partir de l'adoption d'un point de vue impersonnel. La possibilité d'identifier des besoins fondamentaux dépend étroitement d'une théorie morale ${ }^{1}$, en l'occurrence de la distinction entre valeurs relatives à l'agent et valeurs neutres par rapport à l'agent, laquelle recouvre une distinction entre deux points de vue, le point de vue personnel et le point de vue impersonnel ${ }^{2}$. Ce détour par la philosophie morale et la justification éthique permet de fonder en raison l'institution de biens premiers en santé et la priorité de la politique des soins primaires de l'OMS sur toute autre considération de justice globale.

En effet le point de vue impersonnel motive une exigence d'impartialité et d'égalité universelles, tandis que le point de vue personnel fait surgir des motifs et des exigences individualistes, qui font obstacle à la poursuite et à la réalisation de tels idéaux. D'un point de vue impersonnel, le souci impartial du bien de tous est unanimement partagé. L'intuition fondamentale, qui émane de ce point de vue, est que toute vie compte et que nul n'est plus important qu'un autre ${ }^{3}$. Dans cette perspective, la satisfaction de besoins urgents comme le soulagement de la souffrance et des pires privations, l'absence de logement et de vêtement, la sous-nutrition, sans lesquels les individus sont privés de conditions sanitaires minimales, l'accès du plus grand nombre à un niveau de vie d'une décence minimale ainsi qu'à des médicaments de base, revêtent une importance particulière dans la définition d'une résolution acceptable des conflits d'intérêts.

\footnotetext{
${ }^{1}$ Le fait de convoquer la notion d'attente «légitime» renvoie à des principes d'équité et présuppose une théorie morale.

${ }^{2}$ T. Nagel, Egalité et partialité, Paris, PUF, 1991, p. 4.

${ }^{3}$ Dans cette mesure, une priorité doit être accordée aux améliorations concernant la situation et la qualité de vie des individus les plus défavorisés, relativement à celles apportées à la vie des agents les plus favorisés, et cela bien que toute amélioration soit, dans une certaine mesure, évaluée positivement.
} 


\section{b) L'exigence d'un minimum en santé}

La détermination de ce qui est à placer, ou non, du côté des besoins de base ainsi que la quantité de ces derniers, tout à la fois motive et justifie l'idée d'un minimum, imposant un devoir mutuel aux individus de la communauté considérée. Or la santé, à tout le moins, un minimum de santé, en particulier un fonctionnement normal d'une partie de nos facultés - sur lequel nous reviendrons ultérieurement - appartient aux éléments minima requis pour poursuivre une vie humaine ${ }^{1}$.

Précisément, la politique des soins de santé primaires de l'OMS confère une réalité et un contenu à ce «panier de biens» minimal en santé qui constitue un minimum que la collectivité devrait prendre en charge concernant chacun de ses membres. Ces soins, susceptibles de varier d'un pays ou d'une communauté à l'autre, comprennent, comme nous l'avons évoqué, au minimum: la promotion d'une nutrition correcte et d'un approvisionnement convenable en eau saine ; des mesures d'assainissement de base ; la santé maternelle et infantile, y compris la planification familiale ; la vaccination contre les grandes pathologies infectieuses; la prévention et la réduction des endémies locales ; l'éducation concernant les problèmes de santé prédominants et les moyens de les prévenir et de les maîtriser ; le traitement approprié des maladies et des traumatismes courants ${ }^{2}$. En particulier, la politique des soins de santé primaires de l'OMS dresse la liste des paramètres vitaux devant absolument être satisfaits pour qu'une vie décente soit possible. Cette liste, fondée sur la notion de besoin de base, permet de penser et de donner un contenu à un droit que les membres de la communauté pourraient légitimement revendiquer, en l'occurrence un droit à une assistance médicale publique.

c) Pour un droit à la qualité de vie

\footnotetext{
${ }^{1}$ On pourrait ici objecter que les besoins de base, ainsi définis, comportent un élément « conventionnel » important (voir Miller 1976, p. 136-138; Benn et Peters 1959, p. 146 ; Feinberg, 1973, p. 110 ; Wiggins, 1985). Toutefois et quand bien même on admettrait que la ligne de partage entre besoins de base et besoins non élémentaires peut changer avec l'évolution de la société, la distinction entre ces deux types de besoins résulte toujours d'un raisonnement qui renvoie à la nature humaine et à ses besoins fondamentaux. Les attentes naturelles des personnes, dans une société donnée, peuvent jouer le rôle de norme. Les espérances s'ajustent sur les possibilités, de telle sorte qu'une norme relative à un niveau minimum de vie acceptable - bien qu'approximatif - émerge naturellement. Feinberg distingue ainsi ce qui est requis pour vivre une vie minimalement décente, au regard de normes réalistes dans un lieu et un temps donnés, et ce qui est s'ajoute à ces exigences (J. Feinberg, Social Philosophy, p. 110).

${ }^{2}$ Un équivalent de ce panier de biens premiers en santé pourrait être pensé à l'échelon communautaire. Il s'adapterait au niveau de santé des populations européennes et aux besoins qui y sont déjà satisfaits.
} 
Un panier de biens en santé, garanti à tous, serait précisément constitué des biens qui sont des conditions nécessaires de la vie humaine et de l'actualisation des facultés humaines, et non pas simplement des conditions nécessaires de la réalisation de certains buts, que l'on se donne et que l'on pourrait ne pas choisir. La référence à des besoins fondamentaux justifie la revendication d'un «droit à la qualité de vie » rendue possible par la coopération sociale et qui s'inscrirait dans le prolongement d'une problématique des droits naturels ou d'une Charte sociale des droits fondamentaux.

L'exigence de la santé pour tous, ou la garantie de biens premiers en santé pour tous, revient en effet à assurer une qualité de vie minimum à chacun, dont on sait qu'elle n'est garantie ni au niveau mondial ni au niveau européen. Cet objectif est explicitement évoqué par l'OMS puisque l'approche par les biens premiers en santé «est déterminée par des objectifs sociaux tels que l'amélioration de la qualité de la vie et la prestation d'un maximum de bienfaits sanitaires au plus grand nombre $»^{1}$. Dans cette perspective, il n'est pas seulement question de réduire la mortalité infantile ou la mortalité en couche ${ }^{2}$ mais aussi de penser l'accès à la santé comme qualité de vie - à travers l'accès à l'eau potable par exemple. Ce type de préoccupation est totalement absent des textes européens. Le plus récent d'entre eux, le « Deuxième programme d'action communautaire dans le domaine de la santé » ${ }^{3}$, se donne en effet pour objectifs $(\alpha)$ d'améliorer la sécurité sanitaire des citoyens, $(\beta)$ de promouvoir la santé, y compris la réduction des inégalités en la matière, $(\gamma)$ de produire et de diffuser des informations et des connaissances en matière de santé. Pourtant les éléments d'analyse précédemment proposés suggèrent, concernant la détermination conceptuelle des besoins de base et les enjeux éthiques qui y sont liés, que la question de la satisfaction des besoins

\footnotetext{
${ }^{1}$ «Système des politiques de santé de l'OMS. Les soins de santé primaires », p. 4. Or ce type d'exigence ne figure dans aucun des textes de l'Union Européenne évoquant la question de la santé. Ainsi par exemple le premier alinéa du projet de Constitution européenne, article III-278, mentionne qu' « un niveau élevé de protection de la santé humaine est assuré dans la définition et la mise en œuvre de toutes les politiques et actions de l'Union. L'action de l'Union, qui complète les politiques nationales, porte sur l'amélioration de la santé publique, ainsi que la prévention des maladies et affections humaines, et des causes de danger pour la santé physique et mentale. Cette action comprend également: a) la lutte contre les grands fléaux, en favorisant la recherche sur leurs causes, leur transmission et leur prévention ainsi que l'information et l'éducation en matière de santé ; b) la surveillance de menaces transfrontières graves sur la santé, l'alerte en cas de telles menaces et la lutte contre celles-ci. L'Union complète l'action menée par les États membres en vue de réduire les effets nocifs de la drogue sur la santé, y compris par l'information et la prévention ».

2 Comme y fait référence l'objectif 4 du Millénaire : «Réduire la mortalité des enfants de moins de 5 ans », associé à la cible 5 (« Réduire de deux tiers, entre 1990 et 2015 , le taux de mortalité des enfants de moins de 5 ans »). De même, l'objectif 5 est d' " améliorer la santé maternelle » et la cible 6 de "réduire de trois quarts, entre 1990 et 2015, le taux de mortalité maternelle ».

3 Décision $n^{\circ} 1350 / 2007 / C E$ du Parlement européen et du Conseil du 23 octobre 2007 établissant un deuxième programme d'action communautaire dans le domaine de la santé (2008-2013).
} 
premiers en santé gagnerait à être comprise et exploitée en termes de droits à un minimum en santé, y compris au plan global (i.e. au niveau mondial et européen).

La référence à la notion de personne justifie l'interprétation de la problématique des biens premiers en santé, en termes de droits fondamentaux car elle confère un fondement éthique au droit à une qualité de vie minimum, c'est-à-dire, en l'occurrence, au droit à un accès aux biens premiers en santé. La notion de personne est, en effet, un terme de référence de la liste conventionnelle des droits civils, comprenant le droit à la vie, le droit à l'intégrité corporelle, le droit de posséder une voix dans la décision politique, le droit à la liberté de parole, le droit d'association, le droit à la liberté de la presse, le droit à la liberté de culte ${ }^{1}$. Elle confère également un fondement à cette forme de liberté positive, qu'est le droit à un minimum de ressources. La référence à la personne établit ce qui est nécessaire au statut d'humain et donne sens à ce qui est nécessaire à l'épanouissement humain dont l'importance est rappelée par la définition de la santé de l'OMS comme « un état complet de bien-être physique, mental et social $»^{2}$.

\section{Biens premiers en santé et capabilités}

La problématique des soins primaires en santé nous porte au-delà de la question de la santé du corps et du bon fonctionnement de ce dernier, conformément à la définition de la santé proposée par l'OMS et reprise dans la conférence d'Alma-Ata, à laquelle se réfère la politique des soins primaires de santé de l'OMS. La conférence d'Alma-Ata rappelle que « la santé, qui est un complet état de bien-être physique, mental et social et ne consiste pas seulement en l'absence de maladie ou d'infirmité, est un droit fondamental de l'être humain » (article I).

Lorsque l'on mène plus avant l'analyse de la définition des biens premiers en santé, celleci révèle une référence aux capacités, telles qu'Amartya Sen les définit ${ }^{3}$. En effet, l'approche par les capabilités est une façon adéquate d'appréhender le bien-être, d'un point de vue à la

\footnotetext{
${ }^{1}$ Autant de droits que l'on retrouve dans la Charte Sociale des droits fondamentaux, articles 2, 3, 10 à 12.

${ }^{2}$ Si nous avions des droits à tout ce qui est nécessaire à une vie bonne, ces droits seraient trop étendus et se porteraient notamment au-delà des réquisits de santé publique et de la pension de vieillesse. Nous aurions alors droit non pas simplement à des ressources matérielles minima mais à n'importe quel composant insubstituable d'une vie meilleure. C'est pourquoi il convient d'identifier, d'une part, les éléments susceptibles d'augmenter le bien-être et de déterminer, d'autre part, les droits qui peuvent faire l'objet d'une revendication.

${ }^{3}$ Rappelons que pour Sen, la vie peut être mise en équation par un ensemble de «functionings» (qui sont des modes d'être et des faits) en corrélation, tels qu' "être nourri correctement », " être heureux » ou " éprouver du plaisir », «survivre» (voir A. Sen, Commodities and Capabilities, Amsterdam, North-Holland, 1985). L'ensemble des capabilités fournit des informations sur la capacité d'une personne à réaliser son bien-être.
} 
fois subjectif et objectif. Or la mise en évidence de cette référence implicite permet d'asseoir, une nouvelle fois, la primauté d'une politique de santé, comme celle de l'OMS, sur les objectifs proclamés pour le Millénaire. L’introduction de la référence aux capacités dont la satisfaction constitue un objectif des politiques de santé globale renvoie à une interprétation, suggérée par A. Sen, du développement en termes de liberté ${ }^{1}$. En ce sens, réaffirmer la priorité de la politique de soins primaires de l'OMS ne déroge pas à la priorité de la lutte contre la pauvreté visée par le Millénium, dans la mesure où, comme le montre A. Sen, « la pauvreté doit être appréhendée comme une privation des capacités élémentaires, et non, selon la norme habituelle comme une simple faiblesse des revenus $»^{2}$. En outre, la perspective des capacités permet une meilleure compréhension de la pauvreté et des privations, par leur nature et leurs causes « en déplaçant l'examen depuis les moyens [...] vers les fins que les gens ont de bonnes raisons de poursuivre et, conséquemment, vers les libertés mises en jeu pour y parvenir $»^{3}$.

Notre objectif sera donc, dans cette ultime phase de la réflexion, de montrer qu'une définition par les capabilités, des biens premiers en santé, permet d'aller plus loin et d'enrichir la référence aux besoins fondamentaux, d'une part. Nous proposerons ainsi une définition des biens premiers en santé incorporant des capabilités fonctionnelles importantes. Nous montrerons, d'autre part, que la réinterprétation de la politique de l'OMS des soins de santé primaires, à partir de la référence aux capacités, permet, en retour et de surcroît, de contribuer à la priorité conférée à la lutte contre la pauvreté, par les objectifs du Millénium.

\subsection{La satisfaction des capabilités}

Le recours aux capabilités permet en effet d'enrichir la conception des biens premiers en santé, exclusivement centrée sur la notion de besoins fondamentaux. La référence aux capabilités, dans l'analyse des besoins fondamentaux, exploite, dans le domaine de la santé, l'approche proposée par Amartya Sen, dans l'évaluation des états de la société, fondée sur la notion de functionings ${ }^{4}$, c'est-à-dire de performances et d'aptitudes (capabilities) des

\footnotetext{
${ }^{1}$ A. Sen, Un nouveau modèle économique, Paris, Odile Jacob, 2000, p. 15.

${ }^{2}$ A. Sen, Un nouveau modèle économique, p. 123. « La privation de capacités élémentaires se traduit par une mortalité prématurée élevée, de la malnutrition (surtout pour des enfants), une morbidité persistante, un fort taux d'illettrisme et autres problèmes » (A. Sen, Un nouveau modèle économique, p. 36).

${ }^{3}$ A. Sen, Un nouveau modèle économique, p. 126.

${ }^{4}$ Un functioning peut consister dans n'importe quel genre d'action exécutée, ou d'état réalisé, par un individu et peut, a priori, couvrir tout ce qui concerne une description complète de la vie de l'individu. Chaque
} 
individus, telles que le fait d' " être nourri correctement», de «survivre » mais aussi d'être capable de se laver sans aide, de marcher seul, de mener une conversation avec d'autres, d'《être heureux» ou d' "éprouver du plaisir». L'état d'une personne est alors compris comme un vecteur de functionings et l'ensemble des vecteurs faisables constitue l'ensemble des capabilités, c'est-à-dire ce qu'une personne peut faire et ce qu'elle veut faire. Il s'agit, à travers l'approche par les capabilités, de proposer une conception alternative des besoins premiers en santé, fondée sur la capacité de réaliser des functionings, pertinents ou ayant une valeur en tant que tels. Les capabilités fonctionnelles jouent alors un rôle décisif.

Cette approche se justifie en outre à partir de la première perspective que nous avons évoquée dans cette réflexion. Comme nous l'avons vu, la santé est une condition nécessaire pour la jouissance de toute autre consommation. En particulier, les avantages que les personnes retirent de diverses consommations dépendent de leurs capacités d'en extraire du bonheur ou de la satisfaction. Or ces capacités eudémonistiques dépendent aussi de leur santé. La dépression, par exemple, signifie que ces capacités sont très basses. En revanche, diverses autres consommations peuvent être considérées comme étant une prévention de la malnutrition ou de la dépression. La santé renvoie bien à la notion de capacité, au sens que lui donne A. Sen ${ }^{1}$. Cette approche, complémentaire de celle des besoins fondamentaux, permet enfin d'étayer l'appréhension de la dimension objective de la qualité de vie.

Or la justice, au sein de la communauté nationale et, idéalement, internationale et européenne, implique que tous ses membres disposent d'un certain nombre de capacités fondamentales - celle de se nourrir suffisamment, de se mouvoir, de participer à la vie sociale et politique, etc. - selon des modalités et grâce à des moyens, qui peuvent varier d'un contexte socioculturel à un autre. La liste des capabilités fonctionnelles de base, proposée par Martha Nussbaum et qui a une certaine validité transculturelle, est de ce point de vue éclairante. Ces capacités concernent, dans le domaine qui nous intéresse, le fait de : 1 . pouvoir vivre, autant que possible, une vie humaine complète jusqu'à la fin; éviter une mort prématurée $[\ldots]$; 2. pouvoir jouir d'une bonne santé, d'une alimentation adéquate, d'un foyer décent ; avoir des opportunités de satisfaction sexuelle ; pouvoir se déplacer d'un endroit à un

\footnotetext{
functioning désigne l'une des dimensions de ce qui peut importer dans la vie d'une personne, comme par exemple l'absence de maladie, le logement ou la participation économique et sociale.

${ }^{1}$ La notion de capabilité, proposée par A. Sen, permet d'identifier certains besoins « centraux », notamment dans le domaine de la santé. Les capabilités sont, par exemple, essentielles à la détermination des besoins qui doivent être pris en compte, lorsque l'on veut réduire les inégalités, en termes de «qualité de la vie », entre les individus en satisfaisant ces besoins.
} 
autre ; 3. pouvoir éviter toute douleur inutile et connaître l'expérience du plaisir ; 4. pouvoir utiliser nos cinq sens ; pouvoir imaginer, penser et raisonner ${ }^{1}$.

La notion de capabilité donne ainsi lieu à une approche intégrée et complexe des besoins fondamentaux, du bien-être et de la qualité de vie. En effet, priver quelqu'un de la santé, ce n'est pas seulement le laisser en proie à la maladie mais c'est également le priver de liberté. En effet, dès lors que les besoins fondamentaux sont conçus en termes de capabilités, c'est-àdire de possibilités fonctionnelles, ne pas vacciner un enfant contre la poliomyélite, ne consiste pas seulement à le fragiliser immunitairement mais c'est aussi l'empêcher de travailler dans tel ou tel milieu et réduire le champ de ses possibles. De même contracter le virus du SIDA ne signifie pas seulement être malade ou voir son espérance de vie réduite ${ }^{2}$ mais être privé d'une vie sexuelle ordinaire, ne pas pouvoir jouir d'une vie familiale et, si la maladie a été contractée tôt, être mis à l'écart de la vie professionnelle ou de celle de la communauté. En d'autres termes, ce n'est pas seulement de la vie ou de la mort (comme dans le cas du SIDA) dont il est question, lorsque l'on envisage les biens premiers en santé, mais aussi de la possibilité d'avoir une existence humaine accomplie.

Ainsi la problématique de la satisfaction des besoins premiers en santé ne peut être dissociée de la question de la liberté positive (ou liberté réelle) des personnes ${ }^{4}$. La détermination des besoins, par la référence aux capabilités, identifie un ensemble de possibilités fonctionnelles et offre une détermination spécifique de la liberté positive des individus. La référence aux capabilités permet également d'apprécier des aptitudes fonctionnelles, dans le domaine de la santé. Elle élargit le champ couvert par les politiques de santé, grâce à l'attention portée à la liberté réelle des agents.

\subsection{Possibilité fonctionnelles, liberté réelle et politiques de santé}

Appréhender les besoins fondamentaux en termes de capabilités revient à envisager la liberté positive des patients, à partir d'une association caractéristique des capacités internes et

\footnotetext{
1 M. Nussbaum, 2001, «Appendix Nussbaum's List of Capabilities and BHPS Questions Used», "Symposium on Amartya Sen's Philosophy : 5 Adaptive Preferences and Women's Options", Economics and Philosophy, 17, p. 67-88.

${ }^{2}$ L'objectif 6 du Millénium est de « combattre le VIH/sida, le paludisme et d'autres maladies » et la cible 7 corrélée est «d'ici à 2015 avoir stoppé la propagation du VIH/sida et commencé à inverser la tendance actuelle ».

${ }^{3}$ En référence aux capabilités 2 et 3 décrites par M. Nussbaum.

${ }^{4}$ Cette relation se trouve confirmée par les questionnaires sur la qualité de vie, proposés à des personnes subissant des traitements thérapeutiques et montrant que le bien-être mesuré a des liens profonds avec la liberté, en particulier avec la liberté positive.
} 
des chances externes, décrites ensemble comme des possibilités fonctionnelles. Les possibilités fonctionnelles reposent alors sur le pouvoir interne de faire ou de choisir certaines choses et sur la disponibilité des circonstances environnementales et sociales, nécessaires à l'exercice d'une telle puissance. La liberté positive suppose que soient écartées ou diminuées les entraves - externes ou internes -, qui freinent le déploiement des capacités individuelles ${ }^{1}$ puisqu'un individu est moins libre, lorsque certaines alternatives réalisables sont devenues indisponibles et qu'il est non libre, lorsque certaines des solutions faisables ou normalement disponibles s'avèrent être, pour lui, indisponibles. La problématique des besoins fondamentaux, envisagée en termes de capacités, revêt par là un sens à la fois quantitatif et qualitatif. Elle rend alors compte du fait que, comme le suggère A. Sen, la liberté est une composante essentielle du bien-être individuel, conçu de façon non subjective.

Dans la mesure où cette détermination de la liberté et de son évaluation présupposent une compréhension de ce qui constitue la « faisabilité » ou la «normalité » dans les opportunités de choix, il devient possible, de façon ultime, de préciser, en s'appuyant sur une référence à la liberté et aux capabilités, le contenu de ce que Musschenga identifie comme "(le degré de) fonctionnement normal (en tant que membre de l'espèce biologique Homo Sapiens) ou le bien-être objectif $»^{2}$ que l'on trouve dans la définition de la santé par l'OMS. L'information sur la « liberté-de-choix » peut être utilisée comme reflétant les besoins réels individuels et les besoins associés aux capabilités des agents. De la sorte, elle permet de préciser la notion de «fonctionnement normal» de l'individu humain ${ }^{3}$. L'identification de ce degré de « fonctionnement normal » pourra ensuite être utilement mise en œuvre dans l'évaluation des états de santé et dans l'appréciation du bien-être des personnes concernées. Par là, se dessinent les principes d'une évaluation des biens premiers en santé et de la qualité de vie, associant des critères objectifs (les besoins fondamentaux) et des critères subjectifs (l'appréciation, par l'individu, de sa situation personnelle), l'une des façons de prendre en compte ces derniers étant d'évaluer la liberté interne et la liberté externe de l'individu par une liste de capabilités.

\footnotetext{
${ }^{1}$ T. H. Green, Prolegomena to Ethics, Oxford, Clarendon; " On the Different Senses of 'Freedom' as Applied to Will and Moral Progress of Man », in Works of T. H. Green, vol. 2 ; Lectures of the Principles of Political Obligation, Cambridge, Cambridge University Press. S. I. Benn et W. L. Weinstein, par exemple, proposent une conception de la liberté comme non restriction des options, enrichissant la détermination de la liberté négative comme simple absence d'entrave (S. I. Benn et W. L. Weinstein [1971] « Being Free to Act and Being a Free Man », Mind, 80).

${ }^{2}$ A. W. Musschenga [1994], « Quality of Life and Handicapped People », in Nordenfelt [1994], p. 192.

${ }^{3}$ La liberté de choix a une importance intrinsèque au titre de composante de la vie humaine aussi bien que de paramètre du bien-être. La préservation de sa capacité de choix, pour une personne, a une valeur subjective intrinsèque au-delà de sa valeur instrumentale en ce qui concerne ses préférences présentes et réelles.
} 
L'interprétation des besoins fondamentaux en terme de capabilités permet alors de proposer une redéfinition des politiques de santé, à quel que niveau que ce soit, et des objectifs prioritaires de justice globale. Il revient, dans cette perspective, à ces politiques de favoriser, d'une part, la liberté externe de choix des individus, en fournissant des biens personnels appropriés ou des biens collectifs à des prix raisonnables, mais également de favoriser, d'autre part, la liberté interne de choix des personnes, par des traitements ou des actions préventives, qui aident les individus à retrouver ou à préserver leur aptitude personnelle à s'engager dans certaines activités, quand les conditions sociales et environnementales nécessaires sont réunies. Cette démarche contribue, dans le même temps, à promouvoir le développement comme souci de garantir à chacun des libertés réelles.

\section{Conclusion}

Aussi généreux soient les objectifs que se proposent de réaliser, pour le Millénaire, les Etats membres des Nations Unies, nous ne pouvons en attendre la solution aux questions de justice globale qui se posent dans le domaine de la santé. En premier lieu, il est peu probable que la santé ne puisse rivaliser avec l'objectif de réduction de la pauvreté, évaluée à partir du montant de ressources, disponibles quotidiennement pour chacun, au titre d'objectif premier du Millénaire, mais il apparaît en outre que les priorités en santé énumérées par cette Déclaration ne sont pas des plus pertinentes. Une politique fondée sur la garantie à chacun de biens premiers en santé semble, en revanche, plus appropriée, en particulier si celle-ci fait explicitement référence à une liste de capabilités bien choisies, en l'occurrence ces capacités qui contribuent au déploiement de la liberté interne des agents et à la satisfaction des libertés réelles de chacun. De ce point de vue et si tel était le cas, ce type de politique participerait aussi, de façon décisive, à une réduction significative de la pauvreté. Autrement dit, il nous semble possible de réaliser l'objectif phare du Millénium par d'autres voies que celles préconisées, dans cette Déclaration, c'est-à-dire en conférant une priorité fondamentale à la santé et en particulier aux soins et biens premiers en santé, envisagés comme des capacités internes et externes des agents, comme des possibilités fonctionnelles.

De même, si l'Union Européenne entreprenait de se doter d'une réelle politique de santé commune, elle pourrait à profit faire fond sur la référence aux capacités fonctionnelles. Il ne s'agirait alors pas seulement de «lutter contre les grands fléaux transfrontiers » ou de combattre le tabac ou l'abus d'alcool (Projet de Constitution Européenne, Article-III-278, 5.), 
mais de s'engager dans une réelle politique de santé publique qui non seulement réduise les écarts en santé mais assure à chacun une qualité de vie minimum et l'accomplissement des capacités qui en sont le substrat.

\section{Bibliographie}

Benn (S.) et Peters (R.) [1959] Social Principles and the Democratic State, Londres, Allen et Unwin.

Benn (S.I.) et Weinstein (W.L.) [1971] « Being Free to Act and Being a Free Man », Mind, 80, p. 194211.

Feinberg (J.) [1973] Social Philosophy, Englewood Cliffs, Prentice-Hall.

Green (T. H.) [1906] Prolegomena to Ethics, Oxford, Clarendon Press ; " On the Different Senses of 'Freedom' as Applied to Will and Moral Progress of Man », in Works of T. H. Green, vol. 2.

Griffin (J.) [1986] Well-being. Its Meaning, Measurement and Moral Signifiance, Oxford Clarendon Press.

Kolm (S.-C.) [1985] Le Contrat social libéral, Paris, Presses Universitaires de France.

Kolm (S.-C.) [1987a] « Public Economics », New Palgrave Dictionary in Economics, ed. by J. Eatwell et al., London, Macmillan, p. 1047-1055.

Kolm (S.-C.) [1987b] «The Freedom and Consensus Normative Theory of the State», in P. Koslowski, Tübingen, J. B. Mohr (éd.), Individual Liberty and Democratic Decision-Making : The Ethics, Economics and Politics of Democracy, p. 97-127.

Kolm (S.-C.) [1996] Modern Theories of Justice, Cambridge, MA, MIT Press.

Miller (D.) [1976] Social Justice, Oxford, Clarendon Press.

Musschenga (A. W.) [1994] « Quality of Life and Handicapped People », in Nordenfelt [1994].

Nagel (T.) [1991] Egalité et partialité, Paris, PUF.

Nordenfelt L. (éd.) [1994] Concepts and Measurement of Quality of Life in Health Care, Dordrecht, Kluwer.

Nussbaum (M.) [2001] «Appendix Nussbaum's List of Capabilities and BHPS Questions Used», "Symposium on Amartya Sen's Philosophy: 5 Adaptive Preferences and Women's Options", Economics and Philosophy, 17, p. 67-88.

Sen (A. K.) [1985] Commodities and Capabilities, Amsterdam, North-Holland.

Sen (A. K.) [2000] Un nouveau modèle économique, Paris, Odile Jacob, 2000.

Walzer (M.) [1983] Sphères de la justice, Paris, Seuil, 1997.

Wiggins (D.) [1985] «Claims of Need», in T. Honderich (éd.), Morality and Objectivity, Londres, Routledge et Kegan Paul. 